\title{
CONVERGENCE AND INTEGRABILITY OF TRIGONOMETRIC SERIES WITH COEFFICIENTS OF BOUNDED VARIATION OF ORDER $(m, p)$
}

\author{
VERA B. STANOJEVIC
}

(Communicated by J. Marshall Ash)

ABSTRACT. Let $\{c(n)\}$ be a complex null sequence such that for some integer $m \geq 1$ and some $p \in(1,2]$

$$
\sum_{|n|<\infty}\left|\Delta^{m} c(n)\right|^{p}<\infty \text { and } \sum_{n=1}^{\infty}|\Delta(c(n)-c(-n))| \lg n<\infty .
$$

It is shown that the series

$$
\sum_{|n|<\infty} c(n) e^{i n t}, \quad t \in T=\frac{\mathbb{R}}{2 \pi \mathbb{Z}}
$$

converges a.e. and that the well-known condition $C_{w}$ of J. W. Garrett and C. V. Stanojevic $[4,3]$ implies that the series $(*)$ is the Fourier series of its sum. This generalizes results of W. O. Bray and C. V. Stanojevic [1]. An important consequence of the main result is that $n \Delta c(n)=0(1),|n| \rightarrow \infty$, implies that the condition $C_{w}$ is equivalent to the de la Vallee Poussin summability of partial sums $\left\{S_{n}(c)\right\}$ as conjectured in [8].

\section{INTRODUCTION}

Recently in $[13,12]$ complex sequences of bounded variation of an integer order $m \geq 1$ have been used to study pointwise convergence of trigonometric series and the integrability of $p$ powers $(0<p<1)$ of their sums. A complex sequence $c=\{c(n)\}_{|n|<\infty}=\{c(n)\}$ is of bounded variation of order $m \geq 1$ if

$$
\sum_{|n|<\infty}\left|\Delta^{m} c(n)\right|<\infty,
$$

where $\Delta^{m} c(n)=\Delta\left(\Delta^{m-1} c(n)\right)=\Delta^{m-1} c(n)-\Delta^{m-1} c(n+1)$.

Here we propose to study convergence and integrability of series

$$
\sum_{|n|<\infty} c(n) e^{i n t}, \quad t \in T=\mathbb{R} / 2 \pi \mathbb{Z},
$$

Received by the editors September 4, 1990; presented in parts at the fourth annual meeting of the International Workshop in Analysis and its Applications, June 1990, Kupari, Yugoslavia.

1980 Mathematics Subject Classification (1985 Revision). Primary 42A20, 42A32.

Key words and phrases. Convergence and integrability of trigonometric series, sequences of bounded variation of order $(m, p)$. 
using some techniques from $L^{p}$-space, $1<p \leq 2$. This naturally leads to a generalization of bounded variation of order $m$. Specifically, if for some integer $m \geq 1$ and some real $p \geq 1$

$$
\sum_{|n|<\infty}\left|\Delta^{m} c(n)\right|^{p}<\infty
$$

we say that $\{c(n)\}$ is of bounded variation of order $(m, p)$.

The concept of modified cosine sums, as introduced in [6] and later in [4], is applied to the problem of the Fourier character of the cosine trigonometric series and to $L^{1}$-convergence of cosine Fourier series. In the case of complex coefficients the modified sums are defined as follows [2],

$$
G_{n}(c, t)=\sum_{k=0}^{n}[\Delta c(k)] D_{k}(t)+\sum_{k=0}^{n}[\Delta(c(-k)-c(k))]\left(E_{-k}(t)-1\right),
$$

where $E_{k}(t)=\sum_{j=0}^{k} e^{i j t}$, and $D_{k}$ is the Dirichlet kernel.

The modified sums in $[6,4]$ are defined for even coefficients. To compensate for the lack of evenness in the complex case we shall assume that $\{c(n)\}$ is weakly even (see $[2,11])$, i.e.,

$$
\sum_{n=1}^{\infty}|\Delta(c(n)-c(-n))| \lg n<\infty .
$$

Let $S_{n}(a)=S_{n}(a, t)=a_{0} / 2+\sum_{k=1}^{n} a_{k} \cos k t, t \in(0, \pi]$, where $\left\{a_{n}\right\}$ is a real null sequence of bounded variation. Then $\lim _{n} S_{n}(a, t)=f(t)$ exists in $(0, \pi]$. Notice that $G_{n}(a, t)=S_{n}(a, t)-a_{n+1} D_{n}(t)$. In [3] it is proved that $\left\|f-G_{n}(a)\right\|_{L^{1}(0, \pi)}=\circ(1), n \rightarrow \infty$, is equivalent to the following:

for every $\varepsilon>0$ there exists $\delta(\varepsilon)>0$, independent of $n$, such that

$$
\int_{0}^{\sigma}\left|\sum_{k=n+1}^{\infty}\left(\Delta a_{k}\right) D_{k}(t)\right| d t<\varepsilon, \quad \text { for all } n \text {. }
$$

The purpose of this paper is to study trigonometric series with complex null coefficients of bounded variation of order $(m, p)$, satisfying $(1.3)$ and $(\mathscr{C})$, and by using $L^{p}$-estimates, $p>1$, to show that such series converge a.e. to the integrable limits.

\section{MAIN RESUlT}

For some integer $m \geq 1$ and some $p \geq 1$, let $B V(m, p)$ denote the class of all complex null sequences for which (1.1) holds. Let $\mathscr{C}_{w}$ denote the class of all weakly even complex sequences such that for every $\varepsilon>0$ there exists $\delta(\varepsilon)>0$, independent of $n$, and such that

$$
\int_{|t| \leq \delta}\left|\sum_{k=n+1}^{\infty}[\Delta c(k)] D_{k}(t)\right| d t<\varepsilon, \quad \text { for all } n .
$$

The partial sums of trigonometric series with coefficients $\{c(n)\}$ are denoted by $S_{n}(c)=S_{n}(c, t)=\sum_{|k| \leq n} c(k) e^{i k t}$, and if for some $f \in L^{1}(T), c=\hat{f}$, then the partial sums of the corresponding Fourier series are denoted by $S_{n}(f)=$ $S_{n}(f, t)=\sum_{|k| \leq n} \hat{f}(k) e^{i k t}$. The $L^{1}(T)$-norm is denoted by $\|\cdot\|$.

Our main result is the following: 
Theorem 2.1. Let $\{c(n)\} \in B V(m, p)$. Then

(i) $\sum_{|n|<\infty} c(n) e^{i n t}$ converges a.e.;

(ii) $p \in(1,2]$ and $\{c(n)\} \in \mathscr{C}_{w}$ imply that the series (i) is the Fourier series of its sum $f$.

Proof. To prove (i) assume that for some integer $m \geq 1$ and some $p \geq 1,(1.1)$ holds. The case $p=1$ is resolved in [13]. Suppose $p>1$. Since

$$
\sum_{|n|<\infty}\left|\Delta^{m} c(n)\right|^{p}<\infty
$$

the well-known theorem of F. Riesz [7] guarantees that the series

$$
\sum_{|n|<\infty}\left[\Delta^{m} c(n)\right] e^{i n t}
$$

is the Fourier series of some $h \in L^{q}(T), 1 / p+1 / q=1$. The convergence theorem of R. Hunt [5] says that (2.1) converges a.e.

Consider the identity from [13], for $t \neq 0$

$$
\begin{aligned}
S_{n}(c, t)= & \frac{1}{w^{m}(t)} \sum_{|k| \leq n}\left[\Delta^{m} c(k)\right] e^{i k t} \\
& -\frac{1}{w^{m}(t)} \sum_{j=0}^{m-1} w^{j}(t)\left[\left(\Delta^{m-1-j} c(-n+j)\right) e^{i(-n+j) t}\right. \\
& +\sum_{j=-n}^{-n+m-1} c(j) e^{i j t}-\sum_{j=n+1}^{n+m} c(j) e^{i j t},
\end{aligned}
$$

where $w(t)=1-e^{-i t}$.

Then for $t \neq 0$ it follows that

$$
\lim _{n} S_{n}(c, t)=f(t)
$$

exists a.e.. This completes the proof of (i).

For the proof of (ii) we write

$$
f(t)=\sum_{|n|<\infty} c(n) e^{i n t}, \quad t \in T-\{0\},
$$

in the a.e. sense established in (i). Notice that

$$
\begin{aligned}
G_{n}(c, t) & =\sum_{k=0}^{n}[\Delta c(k)] D_{k}(t)+\sum_{k=0}^{n}[\Delta(c(-k)-c(k))]\left(E_{-k}(t)-1\right) \\
& =S_{n}(c, t)-\left[c(n+1) E_{n}(t)+c(-(n+1))\left(E_{-n}(t)-1\right)\right] .
\end{aligned}
$$

Hence, for $t \neq 0$

$$
\lim _{n} G_{n}(c, t)=f(t) \text { a.e. }
$$

and

$$
f(t)-G_{n}(c, t)=\sum_{k=n+1}^{\infty}[\Delta c(k)] D_{k}\left((t)+\sum_{k=n+1}^{\infty}[\Delta(c(-k)-c(k))]\left(E_{-k}(t)-1\right) .\right.
$$


Using the identity (2.2) we have for $t \neq 0$

$$
\begin{aligned}
f(t)-G_{n}(c, t)= & \frac{1}{w^{m}(t)} \sum_{|k| \geq n+1}\left[\Delta^{m} c(k)\right] e^{i k t} \\
& +\frac{1}{w^{m}(t)} \sum_{j=0}^{m-1} w^{j}(t)\left[\left(\Delta^{m-1-j} c(-n+j)\right) e^{i(-n+j) t}\right. \\
& -\sum_{j=-n}^{-n+m-1} c(j) e^{i j t} \\
& +\sum_{j=n+1}^{n+m} c(j) e^{i j t}+\left[c(n+1) E_{n}(t)-c(-(n+1))\left(E_{-n}(t)-1\right)\right] .
\end{aligned}
$$

Therefore for $\delta>0$ we have

$$
\begin{aligned}
\left\|f-G_{n}(c)\right\| \leq & \int_{|t| \leq \delta}\left|\sum_{k=n+1}^{\infty}[\Delta c(k)] D_{k}(t)\right| d t \\
& +\int_{|t|>\delta} \frac{1}{\left|w^{m}(t)\right|}\left|\sum_{|k| \geq n+1}\left(\Delta^{m} c(k)\right) e^{i k t}\right| d t \\
& +\sum_{j=0}^{m-1}\left(\left|\Delta^{m-1-j} c(-n+j)\right|+\left|\Delta^{m-1-j} c(n+j+1)\right|\right) \\
& \times \int_{|t|>\delta}\left|w^{j-m}(t)\right| d t+\sum_{j=-n}^{-n+m-1}|c(j)|+\sum_{j=n+1}^{n+m}|c(j)|+|c(-(n+1))| \\
& +|c(n+1)| \int_{|t|>\delta}\left|E_{n}(t)\right| d t+|c(-(n+1))| \int_{|t|>\delta}\left|E_{-n}(t)\right| d t \\
& +\sum_{k=n+1}^{\infty}|\Delta(c(-k)-c(k))| \lg k .
\end{aligned}
$$

The only difficult term to estimate in the right-hand side of the above inequality is the second term. Applying first the Hölder inequality and then the HausdorffYoung inequality, we obtain

$$
\begin{aligned}
\int_{|t|>\delta} & \frac{1}{|w(t)|^{m}}\left|\sum_{|k| \geq n+1}\left(\Delta^{m} c(k)\right) e^{i k t}\right| d t \\
& \leq \begin{cases}A_{1} \lg \frac{1}{\delta} \sum_{|k| \geq n+1}|\Delta c(k)|, & m=1, p=1, \\
A_{2} \frac{1}{\delta^{1 / q}}\left(\sum_{|k| \geq n+1}|\Delta c(k)|^{p}\right)^{1 / p}, & m=1,2 \geq p>1, \\
A_{3} \frac{1}{\delta^{m-1 / p}}\left(\sum_{|k| \geq n+1}\left|\Delta^{m} c(k)\right|^{p}\right)^{1 / p}, & m>1,2 \geq p>1,\end{cases}
\end{aligned}
$$

where $1 / p+1 / q=1$, and $A_{1}, A_{2}$, and $A_{3}$ are absolute constants. 
The case $m=1, p=1$ is considered in [4,3] and the case $m=1,2 \geq p>1$ in a different context in [2]. In the general case $m>1$ and $1<p \leq 2$, we have

$$
\begin{aligned}
\left\|f-G_{n}(c)\right\| \leq & \int_{|t| \leq \delta}\left|\sum_{k=n+1}^{\infty}[\Delta c(k)] D_{k}(t)\right| d t \\
& +A_{3} \frac{1}{\delta^{m-1 / p}}\left(\sum_{|k| \geq n+1}\left|\Delta^{m} c(k)\right|^{p}\right)^{1 / p} \\
& +\frac{m}{\delta^{m+1}} \max _{0 \leq j \leq m-1}\left(\left|\Delta^{m-1-j} c(-n+j)\right|+\left|\Delta^{m-1-j} c(n+j+1)\right|\right) \\
& +m\left(\max _{-n \leq j \leq-n+m-1}|c(j)|+\max _{n+1 \leq j \leq n+m}|c(j)|\right) \\
& +|c(-(n+1))|+B_{1}|c(n+1)| \lg 1 / \delta+B_{2}|c(-(n+1))| \lg 1 / \delta \\
& +\sum_{k=n+1}^{\infty}|\Delta(c(-k)-c(k))| \lg k .
\end{aligned}
$$

Given $\varepsilon>0$ there exists $\delta(\varepsilon)>0$ and there exists $n_{0}(\varepsilon)$ such that each term on the right-hand side of the last inequality is less than $\varepsilon / 8$, whenever $n>n_{0}(\varepsilon)$. Hence

$$
\left\|f-G_{n}(c)\right\|<\varepsilon, \quad \text { whenever } n>n_{0}(\varepsilon) .
$$

Since $G_{n}(c)$ is a polynomial, we have $f \in L^{1}(T)$. This concludes the proof that the series $\sum_{|n|<\infty} c(n) e^{i n t}$ is the Fourier series of its sum $f$, i.e., $c=\hat{f}$.

\section{SLOWLY VARYING CONVERGENCE MODULI OF ORDER $m$}

In a recent study [9] of $L^{1}$-convergence of Fourier series, the concept of convergence moduli is introduced. Let $\{c(n)\}$ be a sequence of complex numbers and let

$$
\sum_{|n|<\infty} c(n) e^{i n t}
$$

be the formal trigonometric transform of $\{c(n)\}$. For $p>1$ the convergence modulo of (3.1) is defined as

$$
K_{n}^{p}(c)=\sum_{|k| \leq n}|k|^{p-1}|\Delta c(k)|^{p} .
$$

It is shown in [10] that the trigonometric transforms with regularly varying convergence moduli converge a.e.. A nondecreasing sequence $\{R(n)\}$ of positive numbers is $O$-regularly varying if

$$
\varlimsup_{n} \frac{R([\lambda n])}{R(n)} \text { is finite, for } \lambda>1 .
$$

In particular, a nondecreasing sequence $\{R(n)\}$ of positive numbers is slowly varying if $\lim _{n} R([\lambda n]) / R(n)=1$. A necessary and sufficient condition for $\left\{e^{K_{n}^{p}(c)}\right\}$ to be $O$-regularly varying sequence is that

$$
\varlimsup_{n} \sum_{|k|=n+1}^{[\lambda n]}|k|^{p-1}|\Delta c(k)|^{p} \text { is finite, for } \lambda>1 .
$$


Equivalently, $K_{n}^{p}(c)=\lg R(n)$, for some $O$-regularly varying sequence $\{R(n)\}$ if and only if (3.2) holds.

It is quite natural to extend the concept of convergence moduli to include the sequential behavior of $m$ th differences of coefficients of the trigonometric transforms.

Definition 3.1. Let $\{c(n)\}$ be a sequence of complex numbers, and let (3.1) be its trigonometric transform. The convergence modulo of integer order $m \geq 1$ of (3.1) is defined as

$$
K_{n}^{p}(c, m)=\sum_{|k| \leq n}|k|^{p-1}\left|\Delta^{m} c(k)\right|^{p}, \quad p>1 .
$$

The following lemma is needed in the proof of the main result of this section.

Lemma 3.1. Let $\{c(n)\}$ be a sequence of complex numbers. For some integer $m \geq 1$, some $p>1$, let

$$
\varlimsup_{n}\left[K_{[\lambda n]}^{p}(c, m)-K_{n}^{p}(c, m)\right] \text { be finite, for } \lambda>1 \text {. }
$$

Then $\left\{K_{n}^{p}(c, m)\right\}$ is a slowly varying sequence.

Proof. The case $K_{n}^{p}(c, m)=O(1), n \rightarrow \infty$, is trivial. Assume $K_{n}^{p}(c, m) \rightarrow \infty$, $n \rightarrow \infty$. For $\lambda>1$, consider the identity

$$
\frac{K_{[\lambda n]}^{p}(c, m)}{K_{n}^{p}(c, m)}=1+\frac{\sum_{|k|=n+1}^{[\lambda n]}|k|^{p-1}\left|\Delta^{m} c(k)\right|^{p}}{K_{n}^{p}(c, m)} .
$$

Then

$$
\begin{aligned}
1 & \leq \varlimsup_{n} \frac{K_{[\lambda n]}^{p}(c, m)}{K_{n}^{p}(c, m)} \leq 1+\varlimsup_{n} \frac{\sum_{|k|=n+1}^{[\lambda n]}|k|^{p-1}\left|\Delta^{m} c(k)\right|^{p}}{K_{n}^{p}(c, m)} \\
& =1+\varlimsup_{n} \sum_{|k|=n+1}^{[\lambda n]}|k|^{p-1}\left|\Delta^{m} c(k)\right|^{p} \varlimsup_{n} \frac{1}{K_{n}^{p}(c, m)} \\
& \leq 1+C \lim _{n} \frac{1}{K_{n}^{p}(c, m)} \leq 1,
\end{aligned}
$$

where $C$ is an absolute constant depending on $\lambda$. Hence

$$
\lim _{n} K_{[\lambda n]}^{p}(c, m) / K_{n}^{p}(c, m)=1 .
$$

Although the main theorem in this section is a corollary to Theorem 2.1 , it has an independent interest because it relates the pointwise convergence and the integrability of the sum function to the Tauberian condition (3.4), which, as shown in Lemma 3.1, implies the slowly varying nature of the convergence modulo of order $m \geq 1$.

The following theorem is our main result in this section.

Theorem 3.1. Let $\{c(n)\}$ be a complex null sequence and for some $p \in(1,2]$ and some O-regularly varying sequence $\{R(n)\}$ let

$$
K_{n}^{p}(c, m)=\lg R(n) \text {. }
$$


Then the conclusions of Theorem 2.1 hold.

Proof. It suffices to show that (3.5) implies $\{c(n)\} \in B V(m, p)$. From Lemma 3.1 we know that $\left\{K_{n}^{p}(c, m)\right\}$ is a slowly varying sequence, hence $K_{n}^{p}(c, m)=$ $\circ\left(n^{\alpha}\right), n \rightarrow \infty, \alpha>0$. On the other hand we have the inequality

$$
\sum_{|j| \leq n}\left|\Delta^{m} c(j)\right|^{p} \leq C_{1} \sum_{|j| \leq n} \frac{1}{|j|^{p}} K_{j}^{p}(c, m)+\frac{C_{2}}{|n|^{p-1}} K_{n}^{p}(c, m),
$$

where $C_{1}$ and $C_{2}$ are absolute constants. Since the series on the right-hand side of the above inequality is convergent and the second term in the inequality is $\circ(1), n \rightarrow \infty$, we have that the series on the left-hand side of the same inequality is convergent. This proves that the sequence $\{c(n)\} \in B V(m, p)$. The rest of the proof proceeds as in Theorem 2.1.

The independent interest of Theorem 3.1 is best seen in the following corollary, where condition (3.5) is now replaced by a somewhat stronger condition intrinsic to $\{c(n)\}$.

Corollary 3.1. Let $\{c(n)\}$ be a complex null sequence and for some integer $m \geq$ 1 and some $p \in(1,2]$ let

$$
\frac{1}{n} \sum_{|k| \leq n}|k|^{p}\left|\Delta^{m} c(k)\right|^{p}=O(1), \quad n \rightarrow \infty .
$$

Then the conclusions of Theorem 2.1 hold.

Proof. From the inequality

$$
\begin{aligned}
\sum_{|k| \leq n}\left|\Delta^{m} c(k)\right|^{p} \leq & C_{3} \sum_{|k| \leq n-1} \frac{1}{|k|^{p}}\left(\frac{1}{|k|} \sum_{j=1}^{|k|}|j|^{p}\left|\Delta^{m} c(j)\right|^{p}\right) \\
& +C_{4} \frac{1}{n^{p-1}}\left(\frac{1}{n} \sum_{|k| \leq n}|k|^{p}\left|\Delta^{m} c(k)\right|^{p}\right),
\end{aligned}
$$

where $C_{3}$ and $C_{4}$ are absolute constants, it follows that $\{c(n)\} \in B V(m, p)$, which completes the proof.

In particular, if for some $m \geq 1, n \Delta^{m} c(n)=O(1),|n| \rightarrow \infty$, then (3.6) holds. Hence we have a special case of Corollary 3.1.

The above results and remarks may also be helpful in unravelling the condition $\mathscr{C}_{w}$. It has been conjectured in [8] that for certain classes of sequences $\{c(n)\}$ the condition $\mathscr{C}_{w}$ is equivalent to certain summability methods. To this end we have the following proposition.

Proposition 3.1. Let $\{c(n)\}$ be a complex null sequence such that

$$
n \Delta c(n)=O(1), \quad|n| \rightarrow \infty \text {. }
$$

Then

(i) $\lim _{n} S_{n}(c, t)=f(t)$ a.e.;

(ii) $\{c(n)\} \in \mathscr{C}_{w}$ is equivalent to

$$
\lim _{\lambda \rightarrow 1+} \varlimsup_{n}\left\|\frac{1}{[\lambda n]-n} \sum_{k=n+1}^{[\lambda n]} S_{k}(c)-f\right\|=0 .
$$


The proof of Proposition 3.1 goes along the lines of the proof of Corollary 3.1.

The nature of $\mathscr{C}_{w}$ becomes more transparent due to Proposition 3.1; namely, for the sequences $\{c(n)\}$ satisfying (3.7), the condition $\mathscr{C}_{w}$ is equivalent to de la Vallee Poussin summability (3.8) introduced in [10].

\section{REFERENCES}

1. W. O. Bray and Č. V. Stanojević, Tauberian $L^{1}$-convergence classes of Fourier series I, Trans. Amer. Math. Soc. 275 (1983), 59-69.

2. __ Tauberian $L^{1}$-convergence classes of Fourier series II, Math. Ann. 269 (1984), 469486.

3. J. W. Garrett and Č. V. Stanojević, Necessary and sufficient conditions for $L^{1}$-convergence of trigonometric series, Proc. Amer. Math. Soc. 60 (1976), 68-72.

4. __, On $L^{1}$-convergence of certain cosine sums, Proc. Amer. Math. Soc. 54 (1976), 101105.

5. R. A. Hunt, On the convergence of Fourier series, Proc. S. I. U. Conf. on Orthogonal Expansions, Southern Illinois Univ. Press, Carbondale, 1968, pp. 235-255.

6. C. S. Rees and Č. V. Stanojević, Necessary and sufficient conditions for integrability of certain cosine sums, J. Math. Anal. Appl. 43 (1973), 579-586.

7. F. Riesz, Ubereine Verrallgemeinerung des Parsevalschen formel, Math. Z. 18 (1923), 117124.

8. Č. V. Stanojević, Fourier and Fourier-Stieltjes series with regularly varying convergence moduli, invited address, First annual meeting of the International Workshop in Analysis and its Applications, Kupari (Dubrovnik) Yugoslavia, June 1-10, 1986.

9. __ O-regularly varying convergence moduli of Fourier and Fourier-Stieltjes Series, Math. Ann. 279 (1987), 103-115.

10. __ Structure of Fourier and Fourier-Stieltjes coefficients of series with slowly varying convergence moduli, Bull. Amer. Math. Soc. 19 (1988), 283-286.

11. Č. V. Stanojević and V. B. Stanojevic, Generalizations of the Sidon-Telyakovskii theorem, Proc. Amer. Math. Soc. 101 (1987), 679-684.

12. V. B. Stanojevic, Convergence of Fourier series with complex quasimonotone coefficients of bounded variation of order $m$, J. Math. Anal. Appl. 115 (1986), 482-505.

13. __ On a theorem of P. L. Uljanov, Proc. Amer. Math. Soc. 90 (1984), 370-372.

Department of Mathematics, Southwest Missouri State University, Springfield, MisSOURI 65804 